\title{
Final LSND and KARMEN-2 Neutrino Oscillation Results
}

\author{
Joachim Wolf* for the KARMEN Collaboration \\ Institut für experimentelle Kernphysik, Universität Karlsruhe, \\ Postfoch 3640, 76021 Karlsruhe, Germany \\ E-mail: 'joachim. wolf@hik. fzk.dè
}

Abstract: The neutrino experiment KARMEN, a 56 t scintillation detector at the spallation source ISIS in England, has searched for $\bar{\nu}_{\mu} \rightarrow \bar{\nu}_{e}$ oscillations at a distance of $17.6 \mathrm{~m}$ from the spallation target. Neutrinos were produced from $\pi^{+}$and $\mu^{+}$decay at rest with an endpoint of $52.8 \mathrm{MeV}$ of the neutrino energy. $\bar{\nu}_{e}$ were detected through inverse beta decay on free protons of the scintillator with almost no cosmic ray induced background. Results will be shown for data taken from Feb. 1997 to Mar. 2000, after a major upgrade of the experiment. The LSND experiment ran for 6 years at a distance of $30 \mathrm{~m}$ from the target of LANSCE at Los Alamos and observed an excess of events consistent with $\bar{\nu}_{\mu} \rightarrow \bar{\nu}_{e}$ oscillations. Results from the entire LSND data sample will be presented, which suggest that neutrino oscillations occur with a $\Delta m^{2}$ in the range $0.2-10 \mathrm{eV}^{2} / \mathrm{c}^{4}$ and that at least one neutrino has a mass greater than $0.4 \mathrm{eV} / \mathrm{c}^{2}$. Exclusion limits from KARMEN-2 will be compared to the positive oscillation signal from the LSND experiment and the statistical compatibility will be addressed.

\section{The Experimental Configuration}

\subsection{KARMEN}

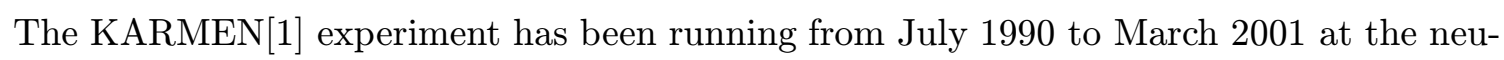
tron spallation facility ISIS of the Rutherford Appleton Laboratory. Neutrinos are produced in ISIS by stopping $800 \mathrm{MeV}$ protons in a massive tantalum target, thereby producing pions. The $\pi^{-}$are absorbed by the target nuclei whereas the $\pi^{+}$decay at rest (DAR). Muon neutrinos $\nu_{\mu}$ therefore emerge from the decay $\pi^{+} \rightarrow \mu^{+}+\nu_{\mu}$. The low momentum $\mu^{+}$are also stopped within the massive target and decay via $\mu^{+} \rightarrow \mathrm{e}^{+}+\nu_{e}+\bar{\nu}_{\mu}$. Because of this $\pi^{+}-\mu^{+}$-decay chain at rest ISIS represents a $\nu$-source with identical intensities for $\nu_{\mu}, \nu_{e}$ and $\bar{\nu}_{\mu}$ emitted isotropically $\left(\Phi_{\nu}=6.37 \cdot 10^{13} \nu / \mathrm{s}\right.$ per flavor for a proton beam current $\underline{\left.I_{p}=200 \mu \mathrm{A}\right) \text {. }}$

\footnotetext{
${ }^{*}$ Speaker.
} 

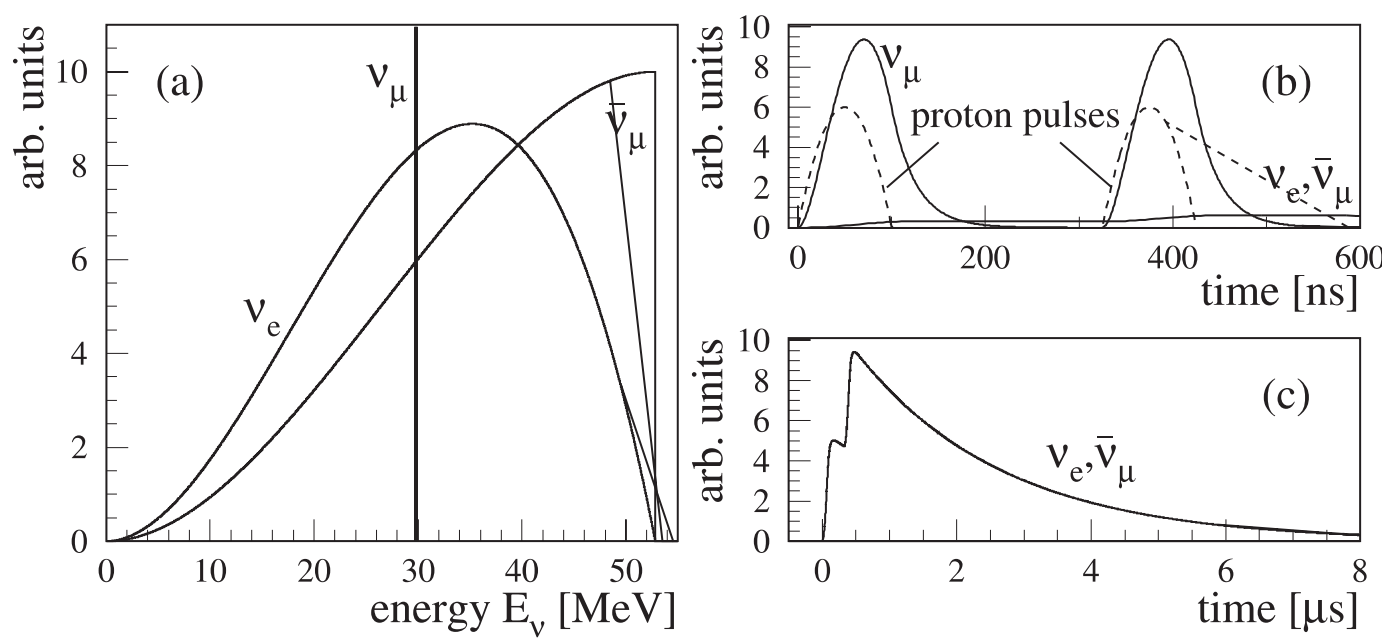

Figure 1: Neutrino energy spectra (a) and production times of $\nu_{\mu}$ (b) and $\nu_{e}, \bar{\nu}_{\mu}$ (c) at ISIS.

A small fraction of $\pi^{-}$decay in flight (DIF), followed by $\mu^{-}$DAR in the target station which again is suppressed by muon capture on high $Z$ nuclei of the spallation target. This decay chain leads to a very small contamination of $\bar{\nu}_{e} / \nu_{e}<6.2 \cdot 10^{-4}\left[\begin{array}{l}3 \\ \mathbf{3}\end{array}\right]$, which is further reduced by software cuts.

The energy spectra of the $\nu$ 's are well defined due to the DAR of both $\pi^{+}$and $\mu^{+}$(Figure $\left.]_{1}^{1} \mathrm{a}\right)$. The $\nu_{\mu}$ 's from $\pi^{+}$-decay are monoenergetic with $\mathrm{E}\left(\nu_{\mu}\right)=29.8 \mathrm{MeV}$, the continuous energy distributions of $\nu_{e}$ and $\bar{\nu}_{\mu}$ up to $52.8 \mathrm{MeV}$ can be calculated using the $\mathrm{V}-\mathrm{A}$ theory. Two parabolic proton pulses of $100 \mathrm{~ns}$ base width and a gap of $225 \mathrm{~ns}$ are produced with a repetition rate of $50 \mathrm{~Hz}$. The different lifetimes of pions $(\tau=26 \mathrm{~ns})$ and muons $(\tau=2.2 \mu \mathrm{s})$ allow a clear separation in time of the $\nu_{\mu}$-burst (Figure $\left.\bar{I}_{1}^{\prime} \mathrm{b}\right)$ from the following $\nu_{e}$ 's and $\bar{\nu}_{\mu}$ 's (Figure $]_{1}^{1} c$ c). The accelerator's duty cycle of $10^{-5}$ allowed effective suppression of cosmic induced background.

The neutrinos were detected in a rectangular tank filled with $56 \mathrm{t}$ of a liquid scintillator [4] at a distance of $17.6 \mathrm{~m}$ from the target. The central scintillation calorimeter was segmented into 512 optically seperated modules. The event position in a module is determined by the time difference of the PM signals at each end of this module. $\mathrm{Gd}_{2} \mathrm{O}_{3}$ coated paper within the module walls provided efficient detection of thermal neutrons due to the very high capture cross section of the $\operatorname{Gd}(\mathrm{n}, \gamma)$ reaction $(\sigma \approx 49000$ barn $)$ in addition to the $\mathrm{p}(\mathrm{n}, \gamma) \mathrm{d}$ capture. The KARMEN electronics had been synchronized to the ISIS proton pulses to an accuracy of better than $\pm 2 \mathrm{~ns}$, so that the time structure of the neutrinos can be exploited in full detail.

A massive blockhouse of $7000 \mathrm{t}$ of steel in combination with a system of two layers of active veto counters provided shielding against beam correlated spallation neutron background, suppression of the hadronic component of cosmic radiation as well as reduction of the flux of cosmic muons. In 1996 an additional third veto counter system with a total area of $300 \mathrm{~m}^{2}$ was installed within the $3 \mathrm{~m}$ thick roof and the $2-3 \mathrm{~m}$ thick walls of the iron shielding [iㅁ] 

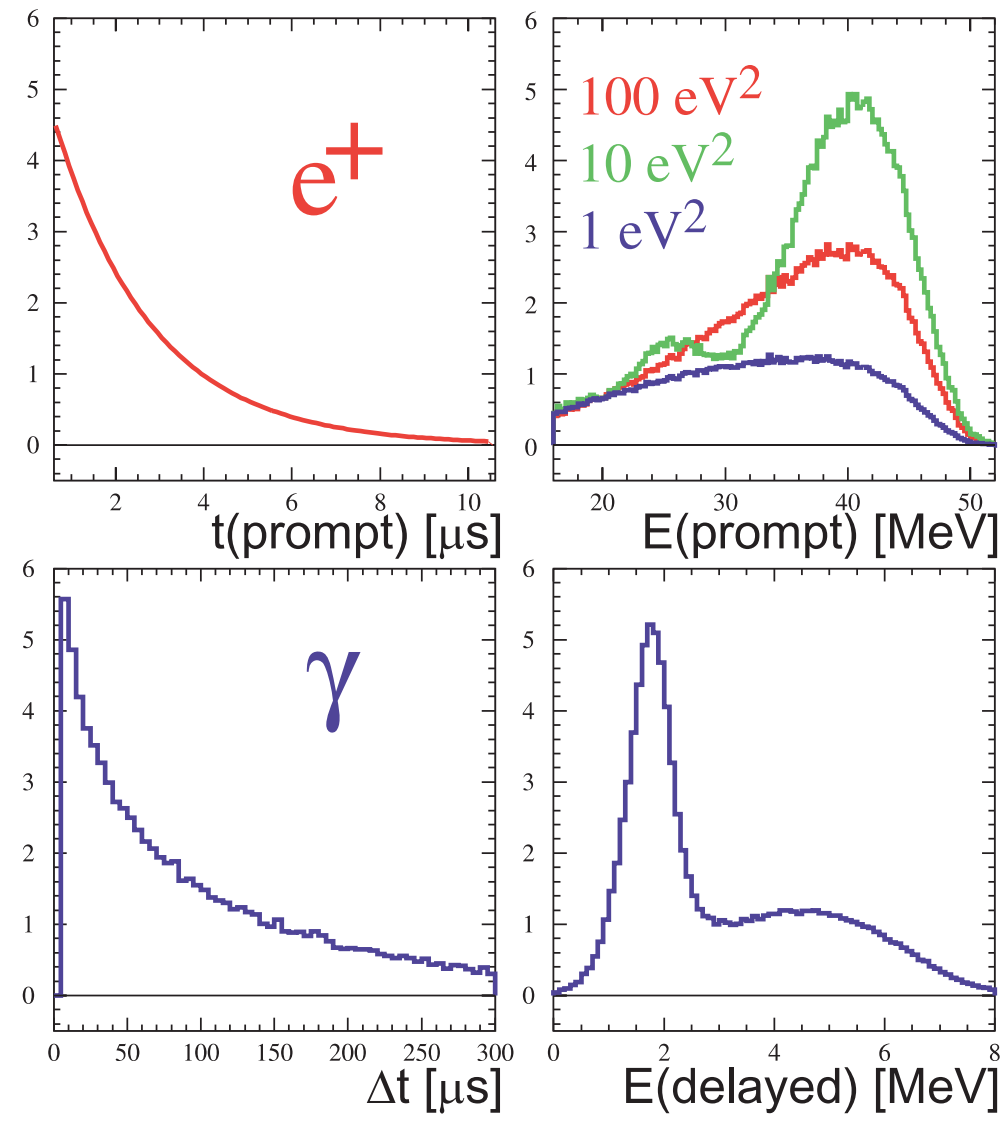

Figure 2: Expected sequences from an oscillation signal: energy and time spectra of $\mathrm{e}^{+}$(simulated) and $\mathrm{n}(\gamma)$ events (measurement of cosmic muon induced neutrons with high statistics)

through the steel at a distance of less than a meter from the main detector and therefore vetoing the successive energetic neutrons from muon deep inelastic scattering, the main background for the $\bar{\nu}_{\mu} \rightarrow \bar{\nu}_{e}$ oscillation search could be reduced by a factor of 40 compared to the KARMEN-1 setup. Results presented in this paper are based on data taken after this upgrade (KARMEN-2).

\subsection{LSND}

The LSND[2] experiment took data over six calender years (1993-1998) at the intense $(1 \mathrm{~mA}) 800 \mathrm{MeV}$ proton beam at the Los Alamos Neutron Science Center (LANSCE), which produced neutrinos from $\pi^{+}$and $\mu^{+}$decay at rest, similar to the neutrino production at ISIS. Due to the different set-up of the target station LSND had a slightly higher contribution $\left(8 \cdot 10^{-4}\right.$ of $\bar{\nu}_{\mu}$ rate) of $\bar{\nu}_{e}$ from $\pi^{-}$and subsequent $\mu^{-}$decay. A small rate $(3 \%)$ of neutrinos from decay in flight could also reach the detector, which was located at a distance of $30 \mathrm{~m}$ downstream of the beam-dump. The pulsed proton beam had a repetition rate of $120 \mathrm{~Hz}$, with a pulse length of $600 \mu \mathrm{s}$.

Cosmic rays were attenuated by $2 \mathrm{~kg} / \mathrm{cm}^{2}$ of overburden. An active veto shield reduced the initial rate of $10 \mathrm{kHz}$ to an acceptable level of $50 \mathrm{~Hz}$. Due to the high duty factor of 
the accelerator the veto shield had to have a very low inefficiency $\left(<10^{-5}\right)$.

Neutrinos were detected in a cylindrical tank filled with $167 \mathrm{t}$ of a dilute solution of liquid scintillator. The low intensity of scintillation light simultaneously allowed to detect Cherenkov light providing information on the direction of the particle. The active area of the inner surface of the tank was $25 \%$, covered by 1220 Hamamatsu 8 " phototubes.

\section{The $\bar{\nu}_{\mu} \rightarrow \bar{\nu}_{e}$ Oscillation Search}

\subsection{KARMEN}

The signature for the detection of $\bar{\nu}_{e}$ is a spatially correlated delayed coincidence of positrons from $\mathrm{p}\left(\bar{\nu}_{e}, \mathrm{e}^{+}\right) \mathrm{n}$ with energies up to $E_{e^{+}}=E_{\bar{\nu}_{e}}-Q=52.8-1.8=51.0 \mathrm{MeV}$ and $\gamma$ emission of either of the two neutron capture processes $\mathrm{p}(\mathrm{n}, \gamma) \mathrm{d}$ with one $\gamma$ of $E(\gamma)=2.2 \mathrm{MeV}$ or $\operatorname{Gd}(\mathrm{n}, \gamma)$ with $3 \gamma$-quanta on average and a sum energy of $\sum E(\gamma)=$ $8 \mathrm{MeV}$ (Figure $\left.{ }_{2}^{\overline{2}}\right)$. The positrons are expected in a time window of several $\mu$ s after beamon-target with a $2.2 \mu$ s exponential decrease due to the $\mu^{+}$decay. The time difference between the $\mathrm{e}^{+}$and the capture $\gamma$ is given by the thermalization, diffusion and capture of neutrons, $\tau_{n} \approx 110 \mu \mathrm{s}$.

The raw data investigated for the oscillation search presented in this paper were recorded in the measuring period of February 1997 through March 2000 which corresponds to $7160 \mathrm{C}$ protons-on-target. The final oscillation analysis will comprise the whole data set with an accumulated neutrino flux corresponding to $\approx 9400 \mathrm{C}$ of protonson-target. The anticipated sensitivity will then reach $\sin ^{2}(2 \Theta)=1.3 \cdot 10^{-3}$ for large $\Delta m^{2}$. A positron candidate is accepted only if there is no previous

\begin{tabular}{lr}
\hline background contribution & events \\
\hline$\left(\mathrm{e}^{-}, \mathrm{e}^{+}\right)$from ${ }^{12} \mathrm{C}\left(\nu_{e}, \mathrm{e}^{-}\right)^{12} \mathrm{~N}_{\text {g.s. }}$ & $3.9 \pm 0.5$ \\
$\nu$ induced random coincidence & $3.5 \pm 0.3$ \\
ISIS $\bar{\nu}_{e}$ contamination & $1.7 \pm 0.2$ \\
cosmic induced sequences & $3.2 \pm 0.2$ \\
\hline total background & $12.3 \pm 0.6$ \\
\hline $\bar{\nu}_{e}$ signal for $\sin ^{2}(2 \Theta)=1$ & $2442 \pm 269$ \\
\hline \hline observed events & 11 \\
\hline
\end{tabular}

Table 1: Expected sequences from different background components within the evaluation cuts specified in the text. Last row: oscillation expectation for maximal mixing. activity in the central detector nor in the two innermost veto counters up to $24 \mu \mathrm{s}$. The required cuts in energy and time for the prompt $(p)$ event are: $0.6 \leq t_{p} \leq 10.6 \mu \mathrm{s}, 16.0 \leq E_{p} \leq 50.0 \mathrm{MeV}$. The cuts on the delayed expected neutron event are as follows: $5.0 \leq t_{d}-t_{p} \leq 300 \mu \mathrm{s}, E_{d} \leq 8 \mathrm{MeV}$ and a volume of $1.3 \mathrm{~m}^{3}$ for the spatial coincidence. Applying all cuts, the total background expectation amounts to $12.3 \pm 0.6$ sequences, where the individual background sources are events induced by cosmic muons, ${ }^{12} \mathrm{C}\left(\nu_{e}, \mathrm{e}^{-}\right){ }^{12} \mathrm{~N}_{\text {g.s. }}$ sequences, $\nu$-induced accidental coincidences and $\left(\mathrm{e}^{+}, \mathrm{n}\right)$ sequences from the intrinsic ISIS $\bar{\nu}_{e}$ contamination.

The individual contributions of the above described background sources are summarized in Table $\underline{11}_{1}^{\overline{1}}$. The last row shows the expectation of $\left(\mathrm{e}^{+}, \mathrm{n}\right)$ sequences from oscillations assuming maximal mixing and $\Delta m^{2} \geq 100 \mathrm{eV}^{2} / \mathrm{c}^{4}$. The background components have been determined precisely during the normal measurements: The $\nu$ induced backgrounds were 

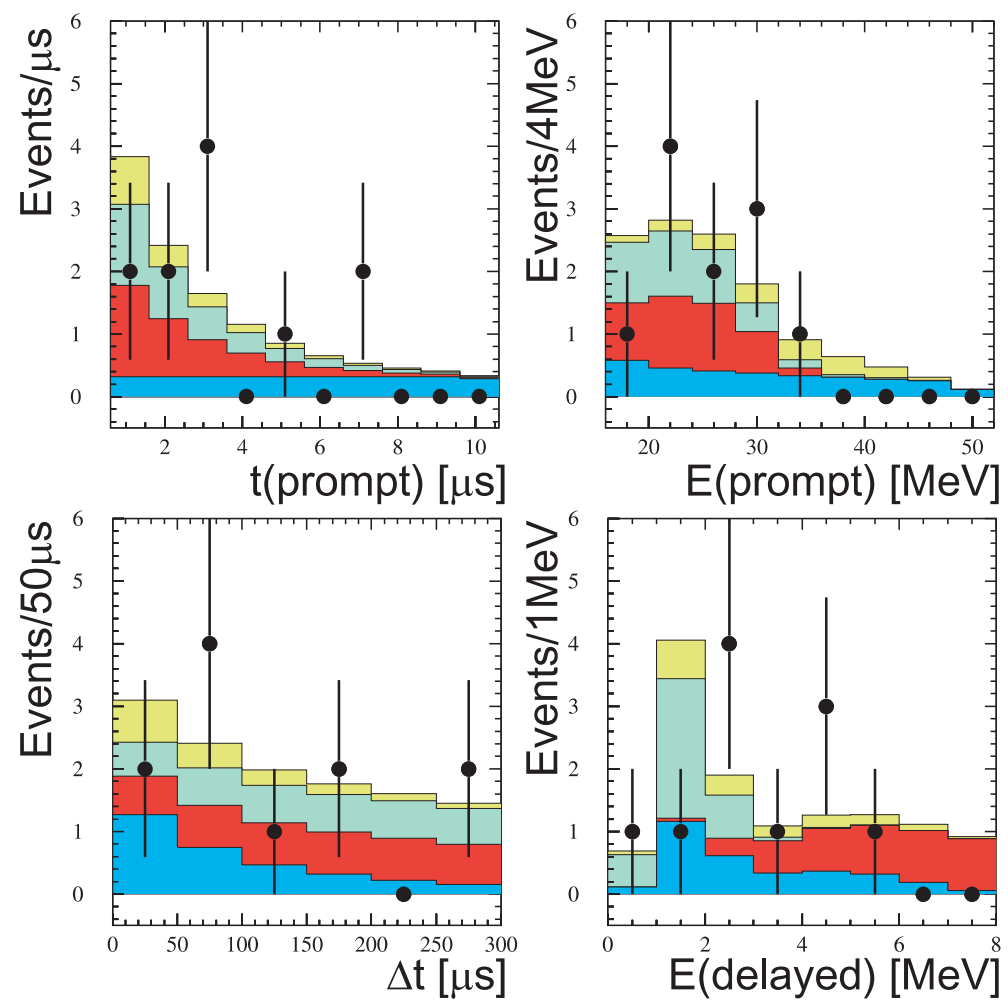

Figure 3: Spectra of the 11 candidate sequences after applying all cuts. Also shown are the background contributions (from bottom to top): cosmic background, $\left(\mathrm{e}^{-}, \mathrm{e}^{+}\right)$from ${ }^{12} \mathrm{C}\left(\nu_{e}, \mathrm{e}^{-}\right){ }^{12} \mathrm{~N}_{\text {g.s. }}$, $\nu$ induced random coincidences and ISIS $\bar{\nu}_{e}$ contamination.

measured with KARMEN in different energy and delayed time windows, the cosmic background was measured with high statistics in the long pre-beam time window. Only the small $\bar{\nu}_{e}$ contribution of the intrinsic source contamination had to be simulated. In addition, the capability of the KARMEN experiment to identify neutrino induced events has been constantly monitored by measuring neutrino-nucleus interactions on ${ }^{12} \mathrm{C}$ via neutral and charged current reactions.

Analyzing the data results in 11 sequential events which satisfy all conditions (see fig-

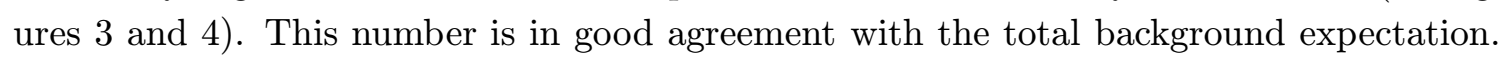
Applying a Bayesian re-normalization of the physically allowed region to the experimental result near a boundary $(N($ osc $) \geq 0,11$ measured events with $12.3 \pm 0.6$ background events expected), an upper limit of $N($ osc $)<6.3$ at $90 \%$ CL can be extracted. An identical procedure had been applied to the initial KARMEN2 data, when the evaluation of a potential oscillation signal was based on a pure counting experiment due to the very small statistics of the background [i $[\overline{1}$ ].

However, with more data and spectral information, a much better evaluation method is a maximum likelihood analysis. Such a likelihood analysis to extract a possible $\bar{\nu}_{\mu} \rightarrow \bar{\nu}_{e}$ signal from these 11 sequences makes use of the precise spectral knowledge of all background sources and a detailed MC description of the oscillation signature in the detector. The 
likelihood function $L$ defined as

$$
L=\prod_{i=1}^{N} f\left(\vec{x}_{i}, \Delta m^{2}, \sin ^{2}(2 \Theta)\right)
$$

is optimized with respect to the free parameters $\Delta m^{2}$ and $\sin ^{2}(2 \Theta)$. The probability density function $f$ is calculated for each of the $N$ event sequences from the parameters $\vec{x}=$ $\left(E_{\text {prompt }}, E_{\text {delayed }}, t_{\text {prompt }}, \Delta t, \Delta \vec{r}\right)$ where $\Delta t$ and $\Delta \vec{r}$ denote the delayed spatial coincidence. The analysis results in a best fit value of oscillation events $N($ osc $)=0$ within the physically allowed range of parameters. As shown in Figure i $\overline{6}_{0}^{\prime}$ an upper limit of 3.8 and 3.1 oscillation events for $\Delta m^{2}<1 \mathrm{eV}^{2} / \mathrm{c}^{4}$ and $\Delta m^{2}>20 \mathrm{eV}^{2} / \mathrm{c}^{4}$, respectively, can be extracted at $90 \% \mathrm{CL}$ based on a complete frequentist approach as suggested by $\left[\overline{i_{i}}\right]$. Assuming maximal mixing $\left(\sin ^{2}(2 \Theta)=1\right), 2442 \pm 269\left(e^{+}, \mathrm{n}\right)$ sequences from oscillations with large $\Delta m^{2}$ were expected. Together with the limit of 3.1 events deduced in the unified frequentist approach, this leads to an upper limit on the mixing amplitude of

$$
\sin ^{2}(2 \Theta)<1.3 \cdot 10^{-3} \quad(90 \% \mathrm{CL})
$$

for $\Delta m^{2} \geq 100 \mathrm{eV}^{2} / \mathrm{c}^{4}$. The corrsponding exclusion curve in $\left(\Delta m^{2}, \sin ^{2}(2 \Theta)\right)$ is given in Fig. $\bar{t}$.

One way of estimating the sensitivity of an experiment is to determine the average limit on the oscillation parameter simulating a large number of experimental outcomes with the actual level of background events, but no oscillation signal. These samples are subsequently analyzed with the same maximum likelihood analysis used for the real data set. The actual KARMEN limit is slightly better than its sensitivity of $\sin ^{2}(2 \Theta)=1.8 \cdot 10^{-3}$ for large $\Delta m^{2}$ and almost identical at lower $\Delta m^{2}$ (see dashed line in Fig. iin). Compared to the ear-

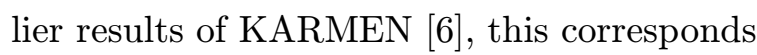
to an improvement of the sensitivity by a factor of $\approx 2.5$.

\subsection{LSND}

The primary search in LSND is for $\bar{\nu}_{\mu} \rightarrow \bar{\nu}_{e}$

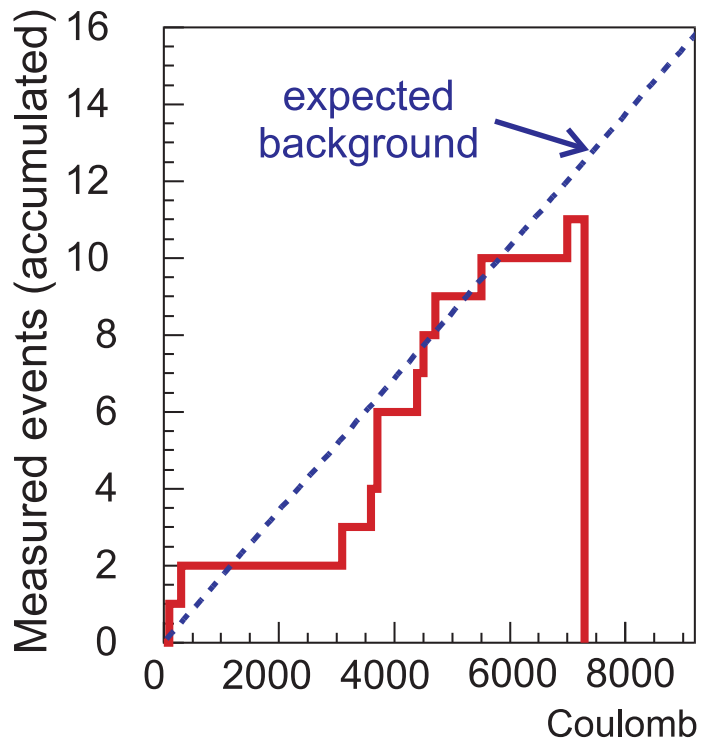

Figure 4: Accumulated oscillation candidates. The dashed line shows the corresponding expected total background.

oscillations with $\bar{\nu}_{\mu}$ from $\mu^{+}$decay at rest.

Like in KARMEN $\bar{\nu}_{e}$ are identified by a $\bar{\nu}_{e}+\mathrm{p} \rightarrow \mathrm{n}+e^{+}$reaction. In addition LSND also accepts $\nu_{\mu} \rightarrow \nu_{e}$ oscillation candidates from $\pi^{+}$decay in flight. The Cherenkov light produced in the LSND scintillator allows to determine the direction of the primary positron with respect to the direction of the incoming neutrino. A subsequent detection of a $2.2 \mathrm{MeV}$ $\gamma$ indicates the capture of a neutron on hydrogen (LSND scintillator contains no Gd). 
Primary positrons are selected choosing $-1.5<\chi_{t o t}^{\prime}<0.5$ where the $\chi_{t o t}^{\prime}$ parameter depends on the product of $\chi$ parameters defined in [1 $\left.1 \overline{1}_{1}^{1}\right]$. Briefly, $\chi_{r}$ and $\chi_{a}$ are quantities minimized for the determination of the event position and direction, and $\chi_{t}$ is the fraction of PMT hits that occur more than $12 \mathrm{~ns}$ after the fitted event time. The chosen energy range $20<E<200 \mathrm{MeV}$ accepts both DAR $\bar{\nu}_{\mu} \rightarrow \bar{\nu}_{e}$ and DIF $\nu_{\mu} \rightarrow \nu_{e}$ oscillation candidates.

Correlated $2.2 \mathrm{MeV} \gamma$ from neutron capture are distinguished from accidental $\gamma$ from radioactivity by use of the likelihood ratio $R_{\gamma}$, which is defined to be the likelihood that the $\gamma$ is correlated divided by the likelihood that it is accidental. $R_{\gamma}$ depends on three quantities: the number of hit PMTs associated with the $\gamma$ (the multiplicity is proportional to the $\gamma$ energy), the distance between the reconstructed positions of the $\gamma$ and positron, and the time interval between the $\gamma$ and positron (neutrons have a capture time in mineral oil of $186 \mu$ s, while accidental $\gamma$ are uniform in time.
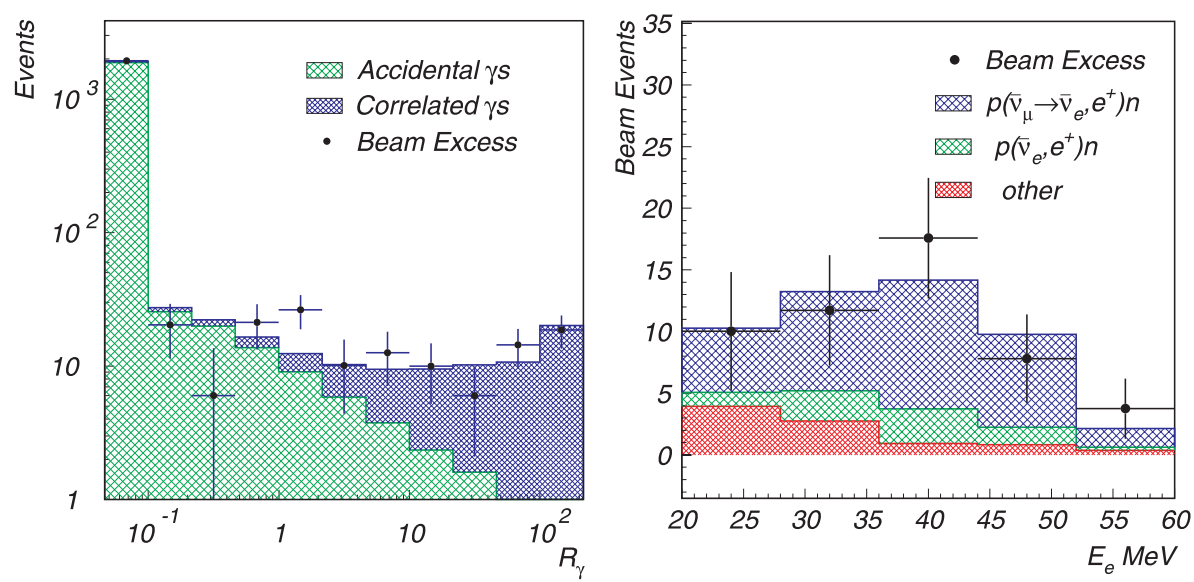

Figure 5: Left: LSND $R_{\gamma}$ distribution for events that satisfy the selection criteria for the primary $\bar{\nu}_{\mu} \rightarrow \bar{\nu}_{e}$ oscillation search. Right: Energy distribution for events with $R_{\gamma}>10$. The shaded region shows the expected distribution from a combination of neutrino background plus neutrino oscillations at low $\Delta m^{2}$

A $\chi^{2}$ fit to the $R_{\gamma}$ distribution, as shown in the left plot of figure off excess of $87.9 \pm 22.4 \pm 6.0$ events after subtracting neutrino background from DAR and DIF. This excess corresponds to an oscillation probability of $(0.264 \pm 0.067 \pm 0.045) \%$. The right plot in figure with $R_{\gamma}>10$.

A likelihood fitter was applied to beam-on events in the final oscillation sample in order to extract the favored oscillation parameters. The likelihood product in the $\left(\sin ^{2} 2 \theta, \Delta m^{2}\right)$ plane is formed over the individual beam-on events that pass the oscillation cuts. This three-dimensional contour is sliced to arrive finally at the LSND allowed region (figure i The number of oscillation events at the best-fit point $\left(\sin ^{2} 2 \theta_{\text {best }}=0.003, \Delta m_{\text {best }}^{2}=1.2 \mathrm{eV}^{2}\right)$ is 89.5 events, which agrees well with the $87.9 \pm 22.4 \pm 6.0$ event excess from the fit to the $R_{\gamma}$ distribution. More details about the final LSND reconstruction and analysis can be found in [i⿱乛龰] oscillations and implies that at least one neutrino has a mass greater than $0.4 \mathrm{eV} / \mathrm{c}^{2}$. 


\section{Comparison}

The limits of KARMEN at 90\% CL and $99 \%$ CL can be compared with the number of oscillation events expected from the final results of the LSND experiment. In Figure $\overline{6}$, the calculated LSND signal strength within the KARMEN detector is shown. For large $\Delta m^{2}$, even at $99 \% \mathrm{CL}$, all of the favored LSND signal range can be excluded by KARMEN2. At lower values of $\Delta m^{2}$, a small fraction of the signal strength of LSND cannot be ruled out by KARMEN2. Note that at high $\Delta m^{2}$ the band of expected events and the exclusion curve are almost parallel. With decreasing values of $\Delta m^{2}$ the expected signal strength becomes smaller. This may be attributed, in part, to the larger source distance for the LSND experiment $\left(\left\langle d_{L S N D}\right\rangle \approx 30 \mathrm{~m}\right.$ compared to $\left.\left\langle d_{K A R M E N}\right\rangle \approx 17.7 \mathrm{~m}\right)$ but may also reflect a smaller signal strength: If the LSND data evaluation yields an oscillation probability $P\left(\bar{\nu}_{\mu} \rightarrow \bar{\nu}_{e}\right)$ which decreases with smaller values of $\Delta m^{2}$-instead of remaining constantthis would have the same effect.

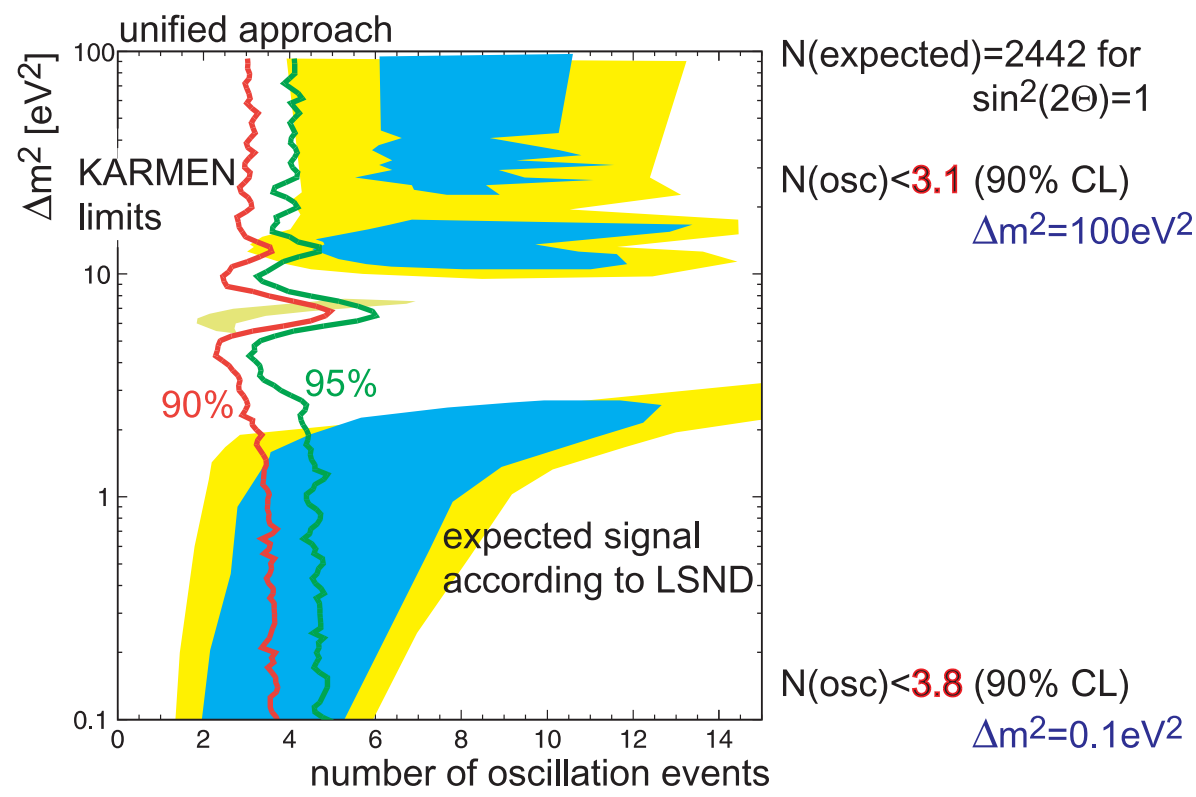

Figure 6: KARMEN exclusion limits at 90\% CL and 99\% CL in comparison with the range of expected oscillation events deduced from the LSND evidence [i. The darker (lighter) region corresponds to logarithmic likelihood values less than 2.3 (4.6) units below the global maximum.

The KARMEN exclusion curve in $\left(\Delta m^{2}, \sin ^{2}(2 \Theta)\right)$ is given in Fig. $\overline{7}_{1}$. Also shown are

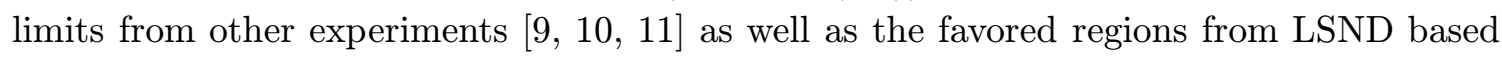
on the complete re-analysis of the entire 1993-98 data set. Again, at high $\Delta m^{2}$, KARMEN excludes the region favored by LSND. At low $\Delta m^{2}$, KARMEN leaves some statistical space, but the reactor experiments at Bugey and Chooz add stringent limits from the $\bar{\nu}_{e}$ disappearance search. Any exclusion curve or favored region is a compactification of complex statistical information, reflecting only a contour of the two-dimensional likelihood functions which have nontrivial properties like multiple side maxima and non-parabolic shapes. Therefore, one needs a quantitative statistical analysis of both LSND and KARMEN based 
on the detailed event-by-event information as demonstrated in [1] sets to deduce correct statements of compatibility or disagreement in terms of frequentist confidence regions.

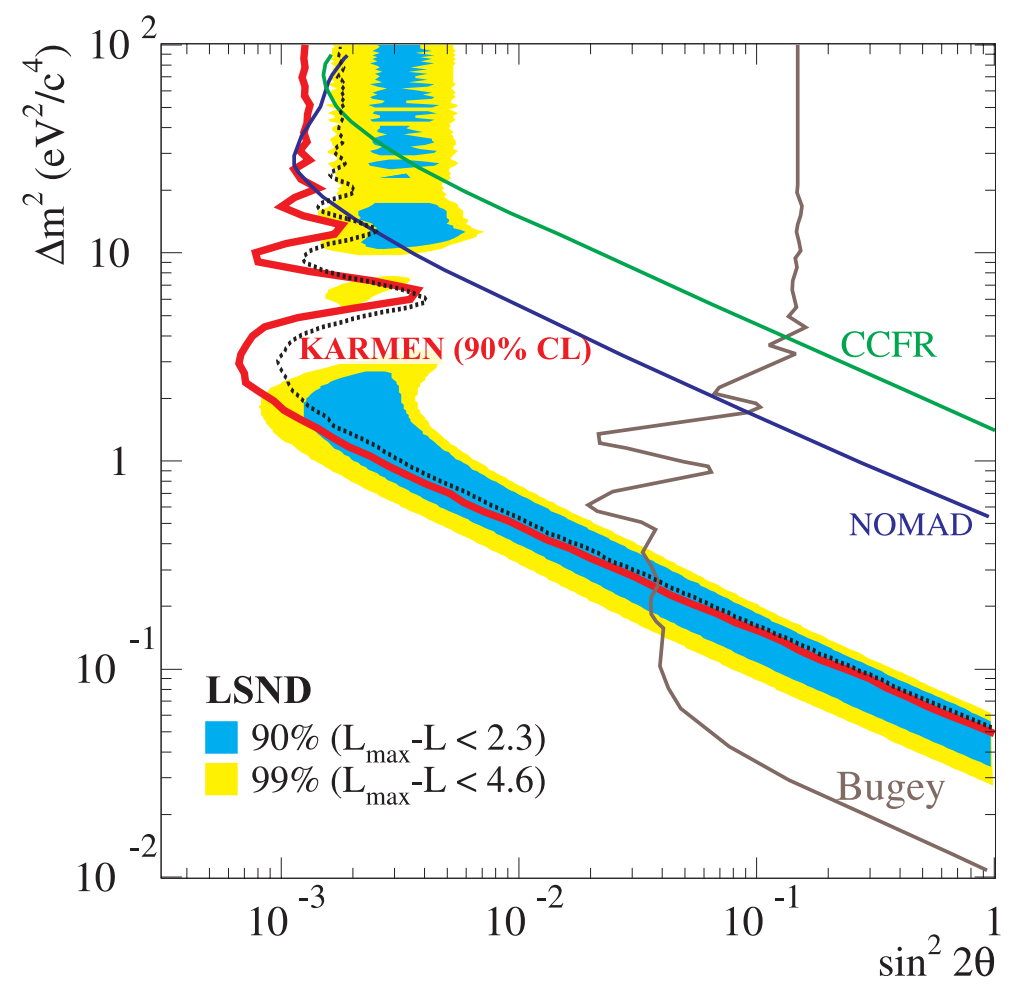

Figure 7: KARMEN2 exclusion limit and sensitivity (dashed line) at 90\% CL compared to other

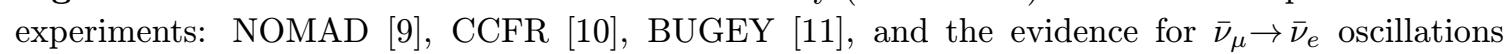
reported by LSND [8]

\section{Conclusions}

KARMEN has stopped data taking in April 2001, having seen no hint for neutrino oscillations so far. LSND found evidence for $\bar{\nu}_{\mu} \rightarrow \bar{\nu}_{e}$ oscillations based on the complete re-analysis of the entire 1993-98 data set. KARMEN and other experiments exclude large sections of the LSND favored region, but leave some statistical space for oscillations. A new effort to cross-check the LSND parameter region is the upcoming BooNE experiment at Fermilab which is under construction. Its sensitivity is expected to improve the final KARMEN sensitivity by another factor of $\left.2[1] \overline{0}{ }_{1}^{i}\right]$ in the appearance mode $\nu_{\mu} \rightarrow \nu_{e}$ with different systematics than KARMEN and LSND.

\section{References}

[1] KARMEN collaboration:

G. Drexlin, K. Eitel, T. Jannakos, M. Kleifges, J. Kleinfeller, C. Oehler, P. Plischke, J. Reichenbacher, M. Steidl, J. Wolf, B. Zeitnitz: Institut für Kernphysik, Forschungszentrum Karlsruhe, Institut für experimentelle Kernphysik, Universität Karlsruhe 
B.A. Bodmann, E. Finckh, J. Hößl, P. Jünger, W. Kretschmer: Physikalisches Institut, Universität Erlangen-Nürnberg

C. Eichner, R. Maschuw, C. Ruf: Institut für Strahlen- und Kernphysik, Universität Bonn

I.M. Blair, J.A. Edgington: Physics Department, Queen Mary and Westfield College, London N.E. Booth: Department of Physics, University of Oxford

[2] LSND collaboration:

E.D. Church, I. Stancu, G.J. VanDalen: University of California, Riverside

W. Vernon: University of California, San Diego

D.O. Caldwell, S. Yellin: University of California, Santa Barbara

D. Smith: Embry Riddle Aeronautical University, Prescott

A. Aguilar, R.L. Burman, J.B. Donahue, G.T. Garvey, W. C. Louis, G.B. Mills, V. Sandberg,

R. Tayloe, D.H. White: Los Alamos National Laboratory, Los Alamos

R. Imlay, A. Malik, W. Metcalf, M. Sung, N. Wadia: Louisiana State University, Baton Rouge

A.K. Cochran, A. Fazely, R.M. Gunasingha, Southern University, Baton Rouge

L.B. Auerbach, R. Majkic: Temple University, Philadelphia

[3] R.L. Burman et al, Nucl. Instrum. Methods A 368, 416 (1996).

[4] G. Drexlin et al, Nucl. Instrum. Methods A 289, 490 (1990).

[5] G. Drexlin et al, Prog. Part. Nucl. Physics 40, 193 (1998).

[6] K. Eitel and B. Zeitnitz, Nucl. Phys. B (proc. suppl.) 77, 212 (1999).

[7] G. Feldman and R. Cousins, Phys. Rev. D 57, 3873 (1998)

[8] A. Aguilar et al., hep-ex/0104049

[9] D. Autiero, talk presented at the 1998 International Conference on High Energy Physics in Vancouver, Canada

[10] A. Romosan et al., Phys. Rev. Lett. 78, 2912 (1997).

[11] B. Achkar et al, Nucl. Phys. B 434, 503 (1995).

[12] M. Apollonio et al, Phys. Lett. B 348, 19 (1995).

[13] K. Eitel, New J. Phys. 2, 1.1 (2000).

[14] C. Athanassopoulos et al, Phys. Rev. C 54, 2685 (1996).

[15] C. Athanassopoulos et al, Nucl. Instrum. Methods A 388, 149 (1997).

[16] A. Bazarko, Nucl. Phys. B (proc. suppl.) 91, 210 (2001). 